\title{
Gradhiva
}

\section{Haïti, première République noire}

Numéro spécial d'Outre-Mer. Revue d'histoire, XC, 340-341, 2003, 366 p.

\section{Colette Pratt}

\section{OpenEdition}

\section{Journals}

Édition électronique

URL : http://journals.openedition.org/gradhiva/413

DOI : $10.4000 /$ gradhiva.413

ISSN : 1760-849X

Éditeur

Musée du quai Branly Jacques Chirac

\section{Édition imprimée}

Date de publication : 1 mai 2005

Pagination : 266-267

ISBN : 2-915133-08-5

ISSN : 0764-8928

Référence électronique

Colette Pratt, « Haïti, première République noire », Gradhiva [En ligne], 1 | 2005, mis en ligne le 10

décembre 2008, consulté le 21 septembre 2020. URL : http://journals.openedition.org/gradhiva/413

DOI : https://doi.org/10.4000/gradhiva.413

Ce document a été généré automatiquement le 21 septembre 2020.

(c) musée du quai Branly 


\section{Haïti, première République noire}

Numéro spécial d'Outre-Mer. Revue d'histoire, XC, 340-341, 2003, 366 p.

\section{Colette Pratt}

\section{RÉFÉRENCE}

Haïti, première République noire, Numéro spécial d'Outre-Mer. Revue d'histoire, XC, 340-341, 2003, 366 p.

1 À l'occasion du deuxième centenaire de l'indépendance d'Haïti, la revue d'histoire Outre-Mer consacre à ce pays un numéro, qui, sous la direction de Marcel Dorigny, regroupe une douzaine d'articles sur l'émergence, l'organisation et la difficile reconnaissance du corps politique haïtien. Le titre du recueil, Haïti, première République noire, appuyé par le graphisme de la couverture, renvoie autant au caractère corporel du politique qu'à la nature politique du corps, toujours inscrit dans des relations de pouvoir. En ce sens on peut dire que tout corps est politique et que chaque régime, chaque société, est un corps dans sa conception spiritualiste ${ }^{1}$. Dans le contexte international du XIX ${ }^{e}$ siècle, Haïti passe pour un corps scandaleux. Les raisons n'en sont pas simples, non plus que les effets d'un tel refus sur l'invention d'une identité haïtienne.

2 L'ambition de penser les tendances du débat qu'illustre ce recueil, m'ont incité à limiter mes commentaires à trois contributions - celle de Dominique Rogers, «De l'origine du préjugé de couleur en Haïti » (pp. 83-101) qui traite du groupe des « libres de couleur », celle de Bernard Gainot et Mayeul Macé, «Fin de campagne à Saint-Domingue » (pp. 15-40) sur l'expédition de 1802, et enfin celle de Thierry Lentz qui traite de l'échec colonial du régime consulaire (pp. 41-60). Ces travaux ont retenu mon attention parce qu'ils se situent au moment des deux grandes ruptures constitutives de l'histoire de la nation haïtienne et de ses principaux lieux de tension, c'est-à-dire la rupture avec l'Ancien Régime et avec l'ordre colonial. Je chercherai à déterminer dans quelle mesure ils renouvellent le débat sur Haïti, permettent d'envisager autrement les difficultés de son histoire. Par exemple, parviennent-ils à sortir du registre moral dans leur 
appréciation de l'importance de la "couleur" dans la conscience commune des Haïtiens, pour jeter un regard anthropologique sur ces phénomènes ? À situer l'histoire de la "République noire» dans le champ des relations ouvertes par les grandes révolutions libérales du XVIII ${ }^{\mathrm{e}}$ siècle?

Je commencerai avec l'enquête de D. Rogers sur «l'origine du préjugé de couleur en Haïti », parce qu'elle pose la question de sa force et de sa durabilité, alors que ses justifications premières n'ont plus cours. En dépit de l'intérêt de cette question, qui a de tous temps interpellé les Haïtiens, étonnés de constater sa survivance au-delà de l'esclavage, l'enquête souffre d'une adhésion évidente au principe que la « race » est un fait naturel indubitable, ignorant ainsi le considérable travail idéologique qui lui donne valeur de vérité 2 . Les réponses insuffisantes apportées en fin de parcours à la question, préoccupante encore aujourd'hui : pourquoi accorde-t-on tant d'importance à la couleur des individus? témoignent de cette difficulté. L'auteur cite comme facteurs déterminants la "visibilité » de la couleur, qui en fait une "différence objective », la structure mentale des populations "africaines " accoutumées aux divisions ethniques, la propagande habile des leaders politiques (p. 100). Une appréhension plus probante de la nature des conflits raciaux demanderait peut-être de se pencher sur la démarche de ceux qui les ont expressément associés à l'émergence des démocraties modernes. Alexis de Tocqueville, par exemple, qui inscrit l'explosion du fait racial à l'époque révolutionnaire dans le rapport dialectique de l'Ancien Régime au monde nouveau qui lui fait place. Sans oublier le propriétaire domingois Julien Raimond, dont les observations et l'action militante nous permettent un coup d'œil privilégié sur la version coloniale d'une passion bien moderne, la passion de l'égalités. C'est bien parce que nous ne partageons pas la même représentation du lien social avec l'ordre monarchique que les trajectoires des libres de couleur dans les archives notariales du XVIII ${ }^{\mathrm{e}}$ siècle étonnent aujourd'hui.

4 Les deux articles qui traitent des événements signant la fin de la colonisation française, me semblent ouvrir, de manière différente, de nouvelles pistes. Avec « Fin de campagne à Saint-Domingue » (pp. 15-40), première des contributions au recueil, B. Gainot \& M. Macé, sensibles au fait que derrière les événements il y a des hommes et des femmes, tentent d'évoquer les mondes relationnels dans lesquels les acteurs évoluaient. La lecture critique des motivations exprimées à travers la confrontation de sources diverses - mémoires, correspondances officielles et personnelles, rapports gouvernementaux, biographies et autres récits historiques - fait ressortir, au-delà des bavures de l'armée de Leclerc, de ses nombreuses exactions contre militaires et civils de couleur, les désirs, ambitions, refus qui arrêtent les choix, définissent l'adversaire, redistribuent les cartes. Le propos envisage les explications habituelles de la défaite française - circonstances accidentelles ou nécessité aveugle, tel que le répète Napoléon à Sainte-Hélène - comme autant de symptômes de la logique de l'expédition. Au-delà de la version officielle, il interroge les témoignages de ces acteurs privilégiés que sont les officiers supérieurs quant à la vraie nature du conflit. Guerre raciale, comme le précise le général Kerverseau dans une lettre à son ami le général Lacroix? Ou simple opération de maintien de l'ordre? L'affrontement des "races ", qui autorise tous les abus, radicalise par sa violence les enjeux, fait perdre aux Français un soutien acquis parmi la population locale et pousse les indécis à rejoindre les insurgés, telle est la conclusion qui s'impose aux plus perspicaces, quelle que soit la manière dont ils agissent par ailleurs. 
Car quels choix s'ouvrent à ces officiers qui se rendent compte de l'inefficacité stratégique et tactique de la violence, ou plus rarement de l'inhumanité d'exécutions sommaires de civils que leur seule couleur de peau condamne? Les situations de guerre offrent un terrain d'étude privilégié à qui veut saisir l'homme dans l'éventail de ses choix et les limites de ses possibilités d'action. Faire preuve de trop d'humanité au regard des objectifs déclarés de son commandement revient à s'exposer à de graves accusations et au renvoi définitif en France, où les occasions de monter en grade sont devenues quasiment nulles. La paix en France métropolitaine limitant sévèrement les chances de promotion, la participation au combat reste une des rares avenues ouvertes aux ambitions de carrière. Ainsi les calculs d'intérêt pèsent lourd contre les principes. Les actions du général Salme et du général Clauzel en témoignent. L'opposition du général Salme au projet de rétablissement de l'esclavage lui vaut un prompt renvoi pour fautes graves et sa subséquente destitution. Fort de tels exemples, le général Clauzel, qui avait dans un premier temps désapprouvé la stratégie répressive du féroce général Rochambeau, successeur de Leclerc, finit par s'y rallier afin de ne pas compromettre sa propre carrière. Le plus souvent, le problème ne se pose même pas à la majorité des officiers et des soldats, tant leur est acquise l'idée de la suprématie blanche. De temps à autre, quelque chose d'aussi ténu qu'un air de musique commun à l'un et l'autre camp jette un pont fragile sur l'abîme d'incompréhension et de perplexité, qui caractérise ce genre d'hostilité. Le général Lacroix, le correspondant de Kerverseau, rapporte que, reconnaissant dans la musique des insurgés les mêmes « airs patriotiques » qu'ils avaient eux-mêmes chantés sur les champs de bataille de la Révolution, les combattants français de la Crête-à-Pierrot sont dans le moment pris de doute : «Les regards de nos soldats interrogeaient les nôtres; ils avaient l'air de nous dire : "Nos barbares ennemis auraient-ils raison? Ne serions-nous plus les soldats de la République? Et serions-nous devenus les instruments serviles de la politique ?" ${ }^{4}$ Néanmoins ces affleurements passagers de sensibilité républicaine n'ont pas raison du sentiment dominant que provoque toute pensée raciale; c'est-à-dire l'incapacité à reconnaître dans cet Autre, désigné comme inférieur, des aspirations communes à tous les hommes qui se veulent libres. Plus qu'aucune autre circonstance, affirment Gainot et Macé, l'impensé racial de l'expédition en détermine l'échec du côté français.

6 Aucune attention pourtant à la présence des mêmes complexités du côté haïtien, malgré le rappel qu'il existe là aussi des divisions. Comme si la rupture qui consacre le partage entre adversaires «blancs » et « noirs » n'avait pas procédé d'un arrachement d'autant plus pénible que les acteurs avaient été proches. Comme s'ils n'avaient pas connu les mêmes difficultés à faire coïncider dans leurs contextes respectifs principes et actions $s^{5}$. Tenir compte du double mouvement de cette histoire, n'est-ce pas mieux saisir de part et d'autre le principe de nos divisions historiques et intimes?

Dans « Bonaparte, Haiti et l'échec colonial du régime consulaire » (pp. 41-60), Thierry Lentz brosse un rapide tableau de l'ensemble de la politique coloniale de Bonaparte, dont il souligne les lacunes. Rappelant que cette politique ne se résume pas au seul rétablissement de l'esclavage mais met en œuvre un vaste projet colonial, Lentz plaide pour une considération plus « dépassionnée » ou " froide » de ce projet. Au programme, le rapprochement des historiens du Consulat, qui ont tendance à traiter l'épisode colonial en sous-dossier de la guerre économique avec l'Angleterre, et des historiens coloniaux portés de leur côté à adopter une vue manichéenne des acteurs et des événements (p. 41). Ce rapprochement ne peut, bien sûr, que profiter à la connaissance 
historique et nous aider à mieux tirer les leçons de nos expériences communes et singulières.

Ces remarques trop brèves visent à discerner les tendances qui pourraient sortir l'histoire coloniale des schémas classiques, et non pas à présenter un bilan des contributions au recueil.

\section{NOTES}

1. Merci à Pierre Manent (du Centre R. Aron de l'EHESS), qui a clarifié pour moi la distinction entre une conception mécanique et une conception spiritualiste du corps.

2. Voir entre autres Simon Linstant, Essai sur les moyens d'extirper les préjugés des Blancs contre la couleur des Africains et des sang-mêlés, Paris, Pagnerre, 1841.

3. Voir Alexis de Tocqueville, De la Démocratie en Amérique, in CEuvres, Paris, Gallimard («Bibliothèque de la Pléiade»), et, sur la description que fait Tocqueville de la transformation de l'homme par la démocratie cf. Pierre Manent, Tocqueville et la nature de la démocratie, Fayard, 1993 ("L'Esprit de la Cité ») (1re éd. 1982) ; Julien Raimond, Mémoires [depuis 1784],Archives Moreau de St-Méry, Dépôt des Fortifications, Mémoires généraux n 245.

4. Il s'agit du Général François Marie Périchou de Kerverseau, du général Jean-Baptiste Salme (dit François) et du Général Pamphile de Lacroix (pp. 18, n. 6; 21; 29, n. 27; 33; 39-40.

5. Voir à de sujet, Michel-Rolph Trouillot, «The Three Faces of Sans-Souci: Glory and Silences in the Haïtian Revolution», in M.-R. Trouillot, Silencing the Past: Power and the Production of History, Boston, Beacon Press, 1995, pp. 31-69.

\section{AUTEUR}

\section{COLETTE PRATT}

colette.pratt@laposte.net 